\title{
Zeros of Hurwitz Zeta Functions
}

\section{By Robert Spira}

Abstract. All complex zeros of each Hurwitz zeta function are shown to lie in a vertical strip. Trivial real zeros analogous to those for the Riemann zeta function are found.

Zeros of two particular Hurwitz zeta functions are calculated.

1. Introduction. The Hurwitz zeta function is defined by

$$
\zeta(s, a)=\sum_{n=0}^{\infty}(a+n)^{-s}, \quad 0<a \leqslant 1,(\operatorname{Re} s>1) .
$$

Let $s=\sigma+i$. In this paper, zero-free regions are found for $\zeta(s, a)$ and zeros are calculated for $a=1 / 3,2 / 3,|t| \leqslant 100, \sigma \geqslant-5$.

\section{Zero-Free Region on the Right.}

TheOREM 1. If $\sigma \geqslant 1+a$, then $\zeta(s, a) \neq 0$.

Proof.

$$
\begin{aligned}
|\zeta(s, a)| & \geqslant a^{-\sigma}-\sum_{n=1}^{\infty}(a+n)^{-\sigma}>a^{-\sigma}-(a+1)^{-\sigma}-\int_{1}^{\infty}(a+x)^{-\sigma} d x \\
& =a^{-\sigma}-(a+1)^{-\sigma}-(a+1)^{1-\sigma} /(\sigma-1),
\end{aligned}
$$

which is $\geqslant 0$ provided $(1+1 / a)^{\sigma}>1+(a+1) /(\sigma-1)$. Now, for $\sigma>1$ and $0<a \leqslant 1$, we have easily $(1+1 / a)^{\sigma}>1+\sigma / a$; so the result holds provided

$$
\sigma / a \geqslant(a+1) /(\sigma-1) \text { or } \sigma(\sigma-1) \geqslant a(a+1) .
$$

Assuming $\sigma \geqslant 1+a$, we have $\sigma-1 \geqslant a$, and multiplying these together, we obtain the last inequality; and Theorem 1 is proved.

\section{Zero-Free Region on the Left.}

THEOREM 2. If $|t| \geqslant 1$ and $\sigma \leqslant-1$, then $\zeta(s, a) \neq 0$.

Proof. We use the functional equation. We have:

$$
\begin{aligned}
\zeta(1-s, a) & =2 \Gamma(s)(2 \pi)^{-s}\left\{\cos \left(\frac{\pi}{2} s\right) \sum_{m=1}^{\infty} \frac{\cos 2 \pi m a}{m^{s}}\right. \\
& \left.\quad+\sin \left(\frac{\pi}{2} s\right) \sum_{m=1}^{\infty} \frac{\sin 2 \pi m a}{m^{s}}\right\} \\
& =2 \Gamma(s)(2 \pi)^{-s} \sum_{m=1}^{\infty} \cos \left(\frac{\pi}{2} s-2 \pi m a\right) m^{-s} \\
& =2 \Gamma(s)(2 \pi)^{-s} \cos \left(\frac{\pi}{2} s-2 \pi a\right)\left\{1+\sum_{m=2}^{\infty} \frac{\cos \left(\frac{\pi}{2} s-2 \pi m a\right)}{\cos \left(\frac{\pi}{2} s-2 \pi a\right)} m^{-s}\right\} .
\end{aligned}
$$


Now, for $|t| \geqslant 1$,

$$
\left|\frac{\cos \left(\frac{\pi}{2} s-2 \pi m a\right)}{\cos \left(\frac{\pi}{2} s-2 \pi a\right)}\right| \leqslant \frac{e^{\pi t}+1}{e^{\pi t}-1} \leqslant \frac{e^{\pi}+1}{e^{\pi}-1} \leqslant 1.09
$$

using

Thus,

$$
|\cos (x+i y)|=\left(\cos ^{2} x+\sinh ^{2} y\right)^{1 / 2}
$$

$$
\left|1+\sum_{m=2}^{\infty} \frac{\cos \left(\frac{\pi}{2} s-2 \pi m a\right)}{\cos \left(\frac{\pi}{2} s-2 \pi a\right)} m^{-s}\right| \geqslant 1-1.09(\zeta(\sigma)-1)>0
$$

for $\sigma \geqslant 2$ as $\zeta(2)<1.645$. Since $\Gamma(s),(2 \pi)^{-s}$ and $\cos ((\pi / 2) s-2 \pi a)$ are all nonzero in $|t| \geqslant 1, \sigma \geqslant 2$, Theorem 2 is proved.

\section{The Trivial Zeros.}

THEOREM 3. If $\sigma \leqslant-(4 a+1+2[1-2 a])$ and $|t| \leqslant 1$, then $\zeta(s, a) \neq 0$ except for trivial zeros on the negative real axis, one in each interval $-2 n-4 a \pm 1$, $n \geqslant 1-2 a$.

Proof. We will apply Rouche's theorem. Using (2) and

$$
u(x)=2 \Gamma(s)(2 \pi)^{-s} \cos \left(\frac{\pi}{2} s-2 \pi a\right), \quad v(x)=u(x) \sum_{m=2}^{\infty} \frac{\cos \left(\frac{\pi}{2} s-2 \pi m a\right)}{\cos \left(\frac{\pi}{2} s-2 \pi a\right)} \frac{1}{m^{s}},
$$

and the rectangle with the four vertices $2 n+1+4 a \pm 1 \pm i$, and taking $2 n+4 a \geqslant 2$, we will have that $\zeta(1-s, a)$ and $u(x)$ will have the same number of zeros in the rectangle provided $|u(x)|>|v(x)|$ on the boundary. This will be so provided

$$
\left|\sum_{m=2}^{\infty} \frac{\cos \left(\frac{\pi}{2} s-2 \pi m a\right)}{\cos \left(\frac{\pi}{2} s-2 \pi a\right)} \frac{1}{m^{s}}\right|<1
$$

on the boundary. This is certainly the case along the top and bottom, as was shown in Section 3. On the vertical edges we have

$$
\begin{aligned}
\left|\frac{\cos \left(\frac{\pi}{2}(2 n+4 a+i t)-2 \pi m a\right)}{\cos \left(\frac{\pi}{2}(2 n+4 a+i t)-2 \pi a\right)}\right| \\
=\left\{\left.\frac{\cos ^{2}\left(\frac{\pi}{2}(2 n+4 a)-2 \pi m a\right)+\sinh ^{2} \frac{\pi}{2} t}{1+\sinh ^{2} \frac{\pi}{2} t}\right|^{1 / 2} \leqslant 1,\right.
\end{aligned}
$$

so $|v(x)| \leqslant|u(x)|(\zeta(2)-1)<|u(x)|$ for $\sigma \geqslant 2$. By symmetry, the single zero of $\zeta(s, a)$ in this rectangle must lie on the real axis. This completes the proof of 
TABLE I

Zeros of $\zeta(s, 1 / 3)$

\begin{tabular}{|c|c|c|c|}
\hline $\operatorname{Re}$ & Im & Re & Im \\
\hline-3.356739 & 0.0 & .382710 & 59.883303 \\
\hline-1.411510 & 0.0 & .29727 .1 & 61.558057 \\
\hline .431293 & 0.0 & .087972 & 63.133469 \\
\hline-.159430 & 7.184833 & .457258 & 65.195474 \\
\hline .342658 & 11.431373 & .563711 & 66.783468 \\
\hline .241817 & 15.189346 & .483299 & 69.515697 \\
\hline-.036837 & 17.768793 & -.067285 & 70.180061 \\
\hline .591803 & 20.690440 & .401279 & 72.270589 \\
\hline .193280 & 23.897873 & .328136 & 74.292420 \\
\hline .127972 & 25.706324 & .520017 & 75.643415 \\
\hline .334406 & 28.524914 & .330148 & 77.920206 \\
\hline .429111 & 30.646264 & .495597 & 79.533738 \\
\hline .462075 & 33.643477 & -.147097 & 80.830920 \\
\hline-.147835 & 35.008686 & .579068 & 82.764724 \\
\hline .506383 & 37.571524 & .533603 & 84.515894 \\
\hline .472657 & 39.696042 & .332095 & 86.302724 \\
\hline .151364 & 42.257863 & .185455 & 88.479212 \\
\hline .343298 & 43.633735 & .350208 & 88.904925 \\
\hline .093732 & 46.080690 & .293211 & 91.542684 \\
\hline .612256 & 47.737933 & .414854 & 92.638777 \\
\hline .442315 & 50.224064 & .542324 & 94.468657 \\
\hline .159285 & 52.406133 & .478417 & 96.639483 \\
\hline .140729 & 53.307053 & -.016600 & 97.910995 \\
\hline .580453 & 56.035147 & .316376 & 99.026723 \\
\hline .322000 & 57.568636 & & \\
\hline
\end{tabular}

Theorem 3. For each $a$, a rectangle contained in $|t| \leqslant 1,-3 \leqslant \sigma \leqslant-1$ has to be investigated individually.

5. Zeros of $\zeta(s, 1 / 3), \zeta(s, 2 / 3)$. The zeros were roughly located by tabulating the functions in the strip $-1 \leqslant \sigma \leqslant 2,0 \leqslant t \leqslant 100$. Then the regula falsi method was applied in the neighborhood of each zero. A final check was made by calculating the change of argument around the boundary of the region. The Euler-Maclaurin formula was used throughout. Table I gives the zeros of $\zeta(s, 1 / 3)$ and Table II the zeros of $\zeta(s, 2 / 3)$. There may be an occasional rounding error in the figures given.

Defining $N(a, T)$ to be the number of zeros of $\zeta(s, a)$ in $0<t \leqslant T$, one should be able to prove, using the method of Berndt [1] that

$$
N(a, T)=\frac{T}{2 \pi} \log T-\left(\frac{1+\log (2 \pi a)}{2 \pi}\right) T+O(\log T),
$$


TABLE II

Zeros of $\zeta(s, 2 / 3)$

$\begin{array}{clcc}\text { Re } & \text { Im } & \text { Re } & \text { Im } \\ -4.582225 & 0.0 & .658788 & 60.192874 \\ -2.629836 & 0.0 & .136371 & 62.718934 \\ -.534265 & 0.0 & .694397 & 65.153529 \\ .166871 & 10.821929 & .145692 & 66.578130 \\ .570050 & 16.605888 & .510215 & 69.521528 \\ .002611 & 20.525222 & .799305 & 71.819557 \\ .850931 & 24.340409 & -.085295 & 73.824766 \\ -.113795 & 28.078257 & .459084 & 75.622482 \\ .721490 & 30.792111 & .745029 & 78.673253 \\ .365790 & 34.136686 & .430076 & 79.806836 \\ .460172 & 37.583838 & .163703 & 82.879125 \\ .203952 & 39.160036 & .288050 & 84.291484 \\ .1597874 & 43.008712 & .766662 & 86.328553 \\ .356658 & 45.347383 & .533808 & 88.742453 \\ .127852 & 47.671788 & .014239 & 91.063946 \\ .595766 & 50.633212 & .718618 & 92.638399 \\ .684428 & 52.731898 & .348818 & 94.360457 \\ -.235421 & 55.856118 & .359310 & 97.077760 \\ .775805 & 57.447893 & .742946 & 98.666525\end{array}$

which for $a=1$ reduces to the usual formula for the number of zeros of $\zeta(2)$.

According to a theorem of Davenport and Heilbronn [2], $\zeta(s, 1 / 3)$ and $\zeta(s, 2 / 3)$ will have zeros with real parts $>1$, but none appeared in the present calculations. It would be interesting to have plots of the orbits as $a$ varies, as well as plots of $\operatorname{Re} \zeta(s, r / 3)=0$ and $\operatorname{Im} \zeta(s, r / 3)=0$ for $-1 \leqslant \sigma \leqslant 2,0 \leqslant t \leqslant 100, r=1,2,3$.

Department of Mathematics Michigan State University East Lansing, Michigan $\mathbf{4 8 8 2 4}$

1. B. BERNDT, “The number of zeros for $\zeta^{(k)}(s)$," J. London Math. Soc. (2), v. 2, 1970, pp. 577-580. MR 42 \# 1776.

2. H. DAVENPORT \& H. HEILBRONN, "On the zeros of certain Dirichlet series," $J$. London Math. Soc., v. 11, 1936, pp. 181-185.

3. J. W. S. CASSELS, "Footnote to a note of Davenport and Heilbronn," J. London Math. Soc., v. 36, 1961, pp. 177-184. MR 26 \#3881. 